\title{
Revisión y evaluación de procesos alternativos al horno alto( ${ }^{(\cdot)}$
}

\author{
Alberto Conejo Nava*
}

\begin{abstract}
Resumen Se hace una revisión crítica de los diversos procesos alternativos al horno alto, tanto de procesos de reducción en estado sólido como de procesos de reducción en estado líquido. La comparación de procesos permite identificar ventajas y desventajas de cada uno de ellos así como señalar sus principales retos para poder competir en la misma escala de producción del horno alto, proceso que hasta ahora domina el escenario mundial de producción de unidades de hierro.
\end{abstract}

Palabras clave Reducción directa. Fusión reductora. Horno alto.

\section{Review and evaluation of alternative processes to the blast furnace}

\begin{abstract}
A critical review on the alternative processes to the blast furnace, involving both solid and liquid state reduction is presented. Process comparison allows to identify advantages and disadvantages of each type of processes as well as to highlight the major challenges faced in order to compete with the traditional ironmaking route: the blast furnace, which today rules the production of iron units worldwide.
\end{abstract}

Keywords Direct reduction. Smelting. Blast furnace.

\section{ANTECEDENTES}

La producción de hierro a escala mundial es dominada por un solo proceso: el horno alto. En 1999 se produjeron 788 millones de toneladas de acero, empleando los siguientes materiales ${ }^{[1]}$ :

- 541 millones de toneladas de arrabio.

- 354 millones de toneladas de chatarra.

- 38,6 millones de toneladas de hierro esponja.

El horno alto constituye una instalación emblemática en la siderurgia, cuyos precedentes se remontan, según Rehder ${ }^{[2]}$, a ocho milenios. En su larga historia se han dado desarrollos impresionantes en su diseño y en su operación, siendo ejemplo de ello la evolución del consumo de carbón, tal como se muestra en la tabla I:

El talón de Aquiles del horno alto es el coque. El consumo mundial de carbón, en 250 plantas de coquización, es de 450 millones de toneladas ${ }^{[3]}$, a partir de las cuales se producen 340 millones de toneladas de coque. Además del problema del coque, existe también el problema relacionado con la alta escala de producción de un horno alto
Tabla I. Consumo histórico de carbón en el horno alto

Table I. Carbon consumption in the blast furnace

\begin{tabular}{cc}
\hline Año & C/ Fe, (t) \\
\hline 1540 & 3,9 \\
1600 & 2,6 \\
1650 & 1,9 \\
1700 & 1,5 \\
1750 & 1,2 \\
1965 & 0,80 \\
1975 & 0,63 \\
1997 & $0,40-0,47$ \\
\hline
\end{tabular}

(1-4,5 mtpa), la cual le confiere poca flexibilidad. Para reducir la elevada dependencia del coque se ha producido una intensa revolución tecnológica del proceso de horno alto en los aspectos, complementarios entre sí, de elevación de la temperatura del viento y de inyección de hidrocarburos (principios de los años 60) o de finos de carbón (principios de los años 80).

El proceso alterno que compita con el horno alto (Tabla II), tomando como referencia su volumen de producción, deberá de reunir características especiales, que permitan:

(•) Trabajo recibido el día 28 de enero de 2000 y aceptado en su forma final el día 18 de diciembre de 2000.

$\left(^{*}\right)$ Instituto Tecnológico de Morelia. Av.Tecnológico 1500, Col. Lomas de Santiaguito. 58120 Morelia, Mich., México. aconejo@antares.tecmor.mx 
Tabla II. Características del horno alto

Table II. Blast furnace features

\begin{tabular}{ll}
\hline \multicolumn{1}{c}{ Características } & \multicolumn{1}{c}{ Magnitudes } \\
\hline Volumen trabajo & $5.000 \mathrm{~m}^{3}$ \\
Reductor & Coque \\
Carga & mineral, sinter, pellet, coque \\
Consumo carbón & $400 \mathrm{~kg} / \mathrm{t}$ arrabio \\
Energía & $14,0 \mathrm{GJ} / \mathrm{t}$ \\
Productividad & $2-2,5 \mathrm{t} / \mathrm{m}^{3} \mathrm{~d}$ \\
\hline
\end{tabular}

- Eliminar o depender a un mínimo del coque, empleando materia prima más barata.

- Eliminar la etapa previa de aglomeración de los finos de mineral de hierro.

- Obtener un producto libre de escoria.

- Aumentar el índice de productividad $\left(\mathrm{t} / \mathrm{m}^{3} \mathrm{~d}\right)$.

- Lograr un menor consumo de energía (GJ/t).

- Disminuir los costes de inversión y operación.

- Realizar un mantenimiento fácil y de bajo costo.

- Conseguir una operación fácil, flexible y libre de riesgos.

- Desarrollar un proceso no contaminante del ambiente.

Se dispone, en la actualidad, de una gran cantidad de procesos alternativos al horno alto que permiten obtener hierro. El objetivo de este trabajo es revisar sus características y evaluar sus ventajas. Todos estos procesos se pueden agrupar en dos tipos: procesos de reducción en estado sólido y procesos de reducción en estado liquido (smelting). La comparación de procesos tomará como base de análisis los aspectos enumerados anteriormente.

\section{EL FENÓMENO DE REDUCCIÓN}

La producción de hierro en el horno alto esta dividida en dos etapas principales: reducción principal en estado sólido seguida de fusión, en la cual se logra una reducción complementaria en estado líquido, y carburación. Otro rasgo importante que presenta este proceso es que el gas reductor se genera de manera interna. La velocidad de producción está determinada directamente por la velocidad de reducción de los óxidos de hierro. Si se toman como referencia los fenómenos químicos de los procesos alternativos, éstos no ofrecen nada nuevo.

La reducción en estado sólido se efectúa por medio del carbono sólido y por medio de reduc- tores en fase gaseosa $\left(\mathrm{CO}\right.$ y $\left.\mathrm{H}_{2}\right)$ generados por reacciones químicas. Las reacciones de reducción que involucran reacciones gas-sólido son más rápidas que las reacciones cuyas interfaces son sólidosólido, debido, no sólo a una mayor área de contacto sino también a una mayor velocidad de difusión de especies gaseosas comparada con la difusión en estado sólido.

Las reacciones principales en la reducción de óxidos de hierro en el horno alto son las siguientes:

$$
\begin{gathered}
\mathrm{aFe}_{\mathrm{x}} \mathrm{O}_{\mathrm{y}(\mathrm{s})}+\mathrm{bCO}_{(\mathrm{g})}=\mathrm{mFe}_{\mathrm{z}} \mathrm{O}_{\mathrm{w}(\mathrm{s})}+\mathrm{nCO}_{2(\mathrm{~g})} \\
\mathrm{C}_{(\mathrm{s})}+\mathrm{CO}_{2(\mathrm{~g})}=2 \mathrm{CO}_{(\mathrm{g})} \\
\Delta \mathrm{H}_{(2)}(1.400 \mathrm{~K})=+166,31 \mathrm{~kJ} / \mathrm{mol}
\end{gathered}
$$

Esta última reacción, conocida como reacción de Boudouard o reacción de pérdida de solución, es altamente endotérmica y se promueve por tanto a altas temperaturas, preferentemente en la zona de toberas.

Durante la reducción del mineral en estado sólido operan diversos mecanismos: transferencia de masa entre el gas reductor y el sólido, transferencia de calor, reacciones químicas, así como fenómenos de nucleación y crecimiento de las nuevas fases. Debido a que la reducción de óxidos de hierro no se efectúa en una sola etapa, sino en varias, los diferentes mecanismos señalados actúan de diversa manera dependiendo de las fases especificas involucradas. La wüstita $\left(\mathrm{Fe}_{1-\mathrm{x}} \mathrm{O}\right)$ y la magnetita $\left(\mathrm{Fe}_{3-\mathrm{x}} \mathrm{O}_{4}\right)$ poseen en común, no solo la misma estructura cúbica sino además el mismo tipo de defectos. Son estructuras deficientes en cationes mientras que, por el contrario, la hematita $\left(\mathrm{Fe}_{2} \mathrm{O}_{3-\mathrm{x}}\right)$ posee una estructura romboédrica deficiente en aniones.

\section{PROCESOS DE REDUCCIÓN DIRECTA}

Los procesos de reducción directa se pueden clasificar, de acuerdo con el tipo de reductor, en procesos que utilizan reductor sólido o procesos que utilizan reductor en fase gaseosa.

Ha existido una gran variedad de procesos y, sin embargo, una gran parte no ha alcanzado la etapa comercial. Actualmente, los procesos que emplean reductor gaseoso obtienen el $92 \%$ de la producción mundial de hierro esponja (DRI). La tabla III resume la producción mundial de DRI por proce$\operatorname{sos}^{[4]}$. 
Revisión y evaluación de procesos alternativos al horno alto

Tabla III. Producción mundial de DRI por procesos en 1999

Table III. Worldwide DRI production by processes in 1999

\begin{tabular}{lcclcc}
\hline $\begin{array}{c}\text { Base } \\
\text { Gas }\end{array}$ & mtpa & $\%$ & $\begin{array}{c}\text { Base } \\
\text { Carbón }\end{array}$ & mtpa & $\%$ \\
\hline MIDREX & 25,97 & 67,3 & SL/RN & 1,16 & 3,0 \\
HYLI & 0,87 & 2,3 & CODIR & 0,15 & 0,39 \\
HYL III & 7,94 & 20,6 & DRC & 0,36 & 0,93 \\
FIOR & 0,34 & 0,90 & KINGLOR & 0,03 & 0,08 \\
FINMET & 0,32 & 0,83 & Otros & 1,2 & 3,1 \\
PUROFER & 0,04 & 0,10 & & & \\
Otros & 0,18 & 0,47 & & & \\
\hline Totales & 35,7 & 92,5 & Totales & 2,9 & 7,5 \\
\hline
\end{tabular}

Antes de iniciar la revisión de procesos comerciales, se señalarán dos procesos cuya importancia es, sobre todo, de tipo histórico. El primer proceso de reducción directa fue el proceso HOGANAS (Tabla IV).

El fundamento operativo del proceso comienza por la colocación de los finos de mineral de hierro dentro de tubos cilíndricos ("saggers"), rodeado de capas compuestas por finos de coque y fundente. El material se transporta dentro de un horno de túnel a una temperatura de $1.200^{\circ} \mathrm{C}$. Se genera un producto de alta metalización y, sin embargo, por ser un proceso con un manejo complicado, no es adecuado para la producción a gran escala. Este proceso se emplea en la producción de polvos de hierro utilizados en metalurgia de polvos.

El sueco Martín Wiberg fue excepcionalmente productivo en el terreno de la reducción de minerales de hierro. Fue él quién invento un proceso novedoso (Tabla V) empleando hornos verticales.

Este proceso es antecesor de los procesos modernos tales como el proceso MIDREX.

Tabla IV. Características del proceso HOGANAS

Table IV. Features of HOGANAS Process

\begin{tabular}{lc}
\hline & HOGANAS \\
\hline Inicio & 1908 \\
Desarrollado por & Hoganas, AB, Suecia \\
Tipo de horno & Túnel \\
Reductor & Reducción carbotérmica \\
Óxido de Hierro & Concentrado $(<20 \mu \mathrm{m})$ \\
Energía & $14,6 \mathrm{GJ} / \mathrm{t}$ \\
\hline
\end{tabular}

Tabla V. Características del proceso WIBERG

Table V. Features of WIBERG'S process

\begin{tabular}{ll}
\hline & WIBERG \\
\hline Inicio & 1918 \\
Desarrollado por & M.Wiberg, Suecia \\
Tipo de horno & Vertical \\
Reductor & CO \\
Generación del reductor & Columna de coque \\
Óxido de Hierro & Pelet \\
Energía & $9,5 \mathrm{GJ} / \mathrm{t}$ \\
\hline
\end{tabular}

\subsection{Procesos con reductor en fase gaseosa}

Los procesos con reductor en fase gaseosa, comercialmente más importantes, son el proceso HYL y el proceso MIDREX. Los procesos FIOR y PUROFER operan comercialmente, pero en baja escala.

\subsection{1. $H Y L$}

El proceso HYL cuenta con dos versiones: el proceso HYLI, que actualmente no se comercializa, y el proceso HYLIII. El proceso HYLI era un proceso por cargas (Tabla VI). Fue el primer proceso comercial con éxito mundial.

El gas reductor es producido por reformado catalítico del gas natural, empleando un exceso de vapor de agua, según la reacción:

$$
\mathrm{CH}_{4(\mathrm{~g})}+\mathrm{H}_{2} \mathrm{O}_{(\mathrm{g})}=\mathrm{CO}_{(\mathrm{g})}+3 \mathrm{H}_{2(\mathrm{~g})}
$$

La reacción es endotérmica. El exceso de vapor de agua es utilizado para prevenir la precipitación de carbono y evitar la desactivación del

Tabla VI. Características del proceso HYLI Table VI. Features of HYLI process

\begin{tabular}{ll}
\hline & HYLI \\
\hline Inicio & 1957 \\
Desarrollado por & Hojalata y Lamina, México \\
Tipo de horno & Lecho fijo (Retorta) \\
Reductor & Gas $\left(\mathrm{CO}+\mathrm{H}_{2}\right)$ \\
Reformado de & Gas natural \\
Óxido de Hierro & Pelet \\
Energía & $11-12,5 \mathrm{GJ} / \mathrm{t}$ \\
\hline
\end{tabular}


catalizador. El desarrollo de nuevos catalizadores ha permitido reducir la cantidad de vapor de agua introducida al reformador.

El gas reductor del reformador, después de condensar el vapor de agua, puede contener aproximadamente $75 \% \mathrm{H}_{2}, 15 \% \mathrm{CO}, 6 \% \mathrm{CO}_{2}$ y $4 \%$ $\mathrm{CH}_{4}$. La necesidad de condensar el vapor de agua, en la versión HYLI, requería volver a calentar el gas reductor antes de introducirlo al reactor de reducción. Otra característica era la falta de homogeneidad del producto, típico de un proceso por cargas de naturaleza discontinua.

El proceso HYLIII se anunció comercialmente en 1979. La reducción del mineral de hierro se efectúa en un reactor vertical (Tabla VII).

El gas de salida no se recicla directamente a la unidad de reformado, lo que implica ventajas e inconvenientes con relación al proceso MIDREX. Las ventajas consisten en que la vida del catalizador se incrementa, debido a la menor cantidad de azufre que circula, y el tamaño del reformador puede reducirse, por manejarse un menor volumen, además de la posibilidad de emplear mineral de hierro con mayor contenido de azufre. El gas se recicla después de condensar el vapor de agua y eliminar el $\mathrm{CO}_{2}$. Esta estrategia requiere de equipo adicional.

El horno vertical esta constituido por tres zonas. La primera es la zona de reducción, la segunda una zona denominada isobárica y la tercera la zona de enfriamiento. Los gases se manejan mediante dos circuitos.

En la actualidad, la versión HYLIII se ha sometido a nuevas revisiones cuyos cambios son relevantes. El primero de ellos es la descarga de producto caliente $\left(700^{\circ} \mathrm{C}\right)$ lo que implica la eliminación de la etapa de enfriamiento y una

Tabla VII. Características del proceso HYLIII

Table VII. Features of HYLIII process

\begin{tabular}{ll}
\hline & HYLIII \\
\hline Inicio & 1979 \\
Desarrollado por & Hojalata y Lamina, México \\
Tipo de horno & Vertical \\
Reductor & Gas $\left(\mathrm{CO}+\mathrm{H}_{2}\right)$ \\
Presión & $>4 \mathrm{~atm}$ \\
Reformado de & Gas natural \\
Óxido de Hierro & Pelet \\
Energía & $10,0-11,0 \mathrm{GJ} / \mathrm{t}$
\end{tabular}

reducción del consumo de energía para fundir posteriormente la carga metálica en el HEA. Durante mucho tiempo, se señaló que una de las principales desventajas del hierro esponja es su gran tendencia a reoxidarse al ser extraído del horno. Esta reoxidación no solamente reduce la metalización sino que además genera una gran cantidad de problemas de tipo operativo, debido a la generación de incendios durante el transporte del material a los hornos eléctricos.

Otra mejora a la versión HYLIII fue la eliminación del reformador externo. El reformado del gas natural se efectúa en el interior del horno, utilizando la capacidad catalizadora del hierro metálico recién reducido. Con estas mejoras $\mathrm{HYL}^{[5]}$ argumenta que su proceso es el de menor consumo de energía en la actualidad, señalando un valor de 9,2 $\mathrm{GJ} / \mathrm{t}$.

\subsubsection{MIDREX}

El proceso MIDREX domina la producción mundial de hierro esponja. Este proceso (Tabla VIII) se desarrolló inicialmente, por la empresa Midland Ross Corporation (MRC) de EE. UU.

El bajo consumo de energía señalado en la tabla anterior considera el reformado in-situ y recuperación del calor sensible de los gases. Un 60 $\%$ del gas de salida del horno de reducción se recicla a través de la unidad de reformado. Esta práctica tiene la ventaja de que los gases oxidantes ya no tienen que ser adicionados externamente, además, a diferencia del proceso HYL, al no agregar vapor de agua, la concentración de esta especie en el gas reductor es baja y no requiere de ser sometido a condensación, con lo cual se evita perder energía. El gas reductor de salida contiene

Tabla VIII. Características del proceso MIDREX

Table VIII. Features of MIDREX's process

\begin{tabular}{ll}
\hline & MIDREX \\
\hline Inicio & 1967 \\
Desarrollado por & MRC, EE.UU. \\
Tipo de horno & Vertical \\
Reductor & $95 \%\left(\mathrm{CO}+\mathrm{H}_{2}\right)$ \\
Presión & $>3 \mathrm{~atm}$ \\
Reformado de & Gas natural \\
Oxido de Hierro & Pelet \\
Energía & $9,5-10,5 \mathrm{GJ} / \mathrm{t}$ \\
\hline
\end{tabular}

http://revistademetalurgia.revistas.csic4ezs3 
aproximadamente $55 \% \mathrm{H}_{2}$ y $33 \% \mathrm{CO}$. En este proceso, el azufre contenido en el gas natural en forma de $\mathrm{H}_{2} \mathrm{~S}$ o $\mathrm{COS}$ debe eliminarse para evitar la destrucción del catalizador debido a la formación de NiS. Una estrategia empleada para reducir la concentración de azufre en el gas a reciclar en el reformador consiste en hacer pasar primeramente esa porción del gas por el circuito de enfriamiento, de manera que el hierro metálico absorba el azufre y limpie el gas.

La relación $\mathrm{H}_{2}$ : $\mathrm{CO}$ del gas de entrada varia de 1,5-1,6. El control de esta relación se determina controlando la cantidad de vapor de agua del gas de salida y el grado de enriquecimiento con gas natural, del gas que sale del reformador.

MIDREX ha señalado recientemente ${ }^{[6]}$ que, en 30 años de vida de este proceso, se ha logrado incrementar la velocidad de reducción y el grado de utilización del gas reductor en un $25 \%$. En los años setenta, el proceso empleaba bajas temperaturas para evitar el problema de sinterización de la carga. A mediados de los noventa se introdujo el concepto de pelet recubierto ("maquillado"), con lo que fue posible aumentar la temperatura de reducción hasta $950^{\circ} \mathrm{C}$. Haciendo reaccionar el gas reductor proveniente del reformador con un flujo extra de oxígeno $\left(12-15 \mathrm{Nm}^{3} / \mathrm{t}\right)$, antes de su ingreso al reactor de reducción, es posible incrementar mas la temperatura del gas, favoreciendo la cinética de reducción.

\subsubsection{FIOR}

El proceso FIOR (Fluidized Iron Ore Reduction) emplea hornos de lecho fluidizado para reducir partículas de mineral de hierro sin aglomerar. El gas reductor se produce por reformación catalítica del gas natural y esta compuesto, en su mayor parte, de hidrógeno. La temperatura de reducción es inferior que la de los procesos descritos previamente, del orden de $800^{\circ} \mathrm{C}$. Esta temperatura es posible debido a una mayor área interfacial sólido-gas con el empleo de finos de mineral y altas presiones. El proceso consta de cuatro reactores en serie, el material fluye por gravedad de uno a otro reactor. En el primero se precalienta el material a $870^{\circ} \mathrm{C}$. El producto se somete generalmente a briqueteado (Tabla IX).

Este proceso presenta problemas relacionados con un estricto control de la velocidad de fluidización del gas y alto consumo de energía. La nueva versión de este proceso se denomina FINMET, conservando las características esenciales e incorporando mejores sistemas de control. Se construyen dos plantas de 2 mtpa en Australia y Venezuela.
Tabla IX. Características del proceso FIOR

Table IX. Features of FIOR's process

\begin{tabular}{ll}
\hline & FIOR \\
\hline Inicio & 1971 \\
Desarrollado por & ESSO (EXXON), EE.UU. \\
Tipo de horno & Lecho fluidizado \\
Reductor & Gas $\left(\mathrm{CO}+\mathrm{H}_{2}\right)$ \\
Presión & 10 atm \\
Reformado de & Gas natural \\
Oxido de Hierro & Finos de hierro (-12 mesh) \\
Energía & $16,7 \mathrm{GJ} / \mathrm{t}$ \\
\hline
\end{tabular}

\subsubsection{AREX}

El proceso AREX (Auto, REX), desarrollado en 1987. por Dam y Bueno de la empresa SIDOR en Venezuela, fue el primer proceso a escala industrial en implementar la autoreformación catalítica del gas natural, usando el hierro esponja recién reducido como catalizador y empleando un horno MIDREX. La empresa italiana Danieli comercializa actualmente este proceso, con la denominación DANAREX.

\subsubsection{PUROFER}

Este proceso fue desarrollado por el alemán A. Thyssen a finales de los años sesenta. El gas reductor se produce mediante dos reformadores cuyas paredes están impregnadas con catalizador de níquel. Los reformadores actúan de manera regenerativa, es decir, uno de ellos se calienta a $1.400^{\circ} \mathrm{C}$ con los gases de salida, mientras que en el otro se introduce el gas natural a reformar. Debido al efecto enfriante de la reacción de reformación del gas natural, la temperatura disminuye de 1.400 a 1.200 ${ }^{\circ} \mathrm{C}$, momento en el que el gas reformado se transfiere al segundo reformador para calentarse. Durante la reformación, el azufre del gas natural se deposita sobre el catalizador, pero durante la siguiente etapa de precalentamiento este elemento es oxidado y eliminado en el gas de salida. Este proceso ya no se comercializa.

\subsubsection{NSC}

Este proceso fue desarrollado por Nippon Steel Corporation, siendo similar a los procesos mencionados que emplean hornos verticales. $\mathrm{Su}$ 
característica principal consiste en que el enfriamiento se realiza en un recipiente separado del reactor de reducción. El proceso opera a altas presiones. El proceso no se comercializó, tras fallidos intentos de operar una planta en $\mathrm{Ma}$ lasia.

\subsubsection{CIRCORED}

Proceso desarrollado por la empresa Lurgi de Alemania a fines de los años setenta. Es un proceso de reducción de finos de mineral que emplea lechos fluidizados. El proceso tiene dos etapas: en la primera, los finos de mineral se precalientan y en la segunda, se efectúa la reducción en presencia de hidrógeno y a una presión de operación de 4 bar. El tamaño de partícula es de $4 \mu$.

\subsubsection{IRON CARBIDE}

El proceso IC (Iron Carbide) fue desarrollado, inicialmente, por el sueco Stelling en 1957. En su momento, este proceso tuvo problemas de adherencia de los finos de mineral debido a la formación de óxidos de bajo punto de fusión, combinados con FeO. En 1977, el norteamericano Stephens propuso una estrategia de control para mejorar el proceso, en el cual los finos de mineral de $+0,1 \mathrm{~mm}-1 \mathrm{~mm}$ se precalientan a $700{ }^{\circ} \mathrm{C} \mathrm{y}$, posteriormente, se introduce a un reactor de lecho fluidizado, el cual opera a una presión de 1,8 atm y una temperatura de operación de $570{ }^{\circ} \mathrm{C}$. El gas reactante esta compuesto por $60 \% \mathrm{CH}_{4}, 34 \% \mathrm{H}_{2}$, $2 \% \mathrm{CO}, 1 \% \mathrm{H}_{2} \mathrm{O}$. Este gas de proceso se forma mezclando gas hidrogeno con gas reciclado que ha sido limpiado previamente. La planta piloto de Trinidad y Tobago proyectada para 0,3 mtpa nunca rebasó 0,1 mtpa, desde 1992 a 1999, debido en parte a tiempos de proceso muy prolongados. Qualitech construyo una planta en EE. UU., pero su operación actualmente esta suspendida.

Existe una gran diferencia entre los productos obtenidos en los procesos anteriores y el producto obtenido en el proceso IC. En un proceso convencional de reducción directa, el producto es hierro metálico $\left(\mathrm{Fe}^{0}\right)$. En cambio, en el proceso IC, el producto principal es carburo de hierro $\left(\mathrm{Fe}_{3} \mathrm{C}\right.$ o $\mathrm{Fe}_{5} \mathrm{C}_{2}$ ). La cantidad de carbono en el hierro esponja varia generalmente de $1,5-3 \% \mathrm{C}$. En cambio en el carburo de hierro puede variar de 7,7$10 \% \mathrm{C}$, dependiendo del tipo de carburo producido, constituyendo una fuente de energía química durante su fusión posterior.

\subsection{Procesos con reductor sólido}

Los procesos con reductor en fase gaseosa requieren del suministro de gas natural para producir el gas reductor. Este producto energético es costoso en comparación con el carbón no coquizable. Para reducir costos de energía o bien, si la disponibilidad del gas natural es baja, se cuenta con una serie de procesos que operan con carbón como reductor. Al introducir cantidades elevadas de carbón, se incrementa el azufre, razón por la cual se coloca cal o caliza en la carga para absorber el azufre. El carbón además de actuar como reductor es también la fuente principal de calor.

El reductor sólido principal es el carbono, el cual produce otro agente reductor gaseoso, el CO, responsable del incremento de la presión interna del horno. Cuando actúa el carbono sólido, la reducción se da, únicamente, en los puntos de contacto, actuąndo temporalmente ya que, cuando se forma una capa de hierro metálico, la reducción ya no procede por contacto sino por difusión de átomos de carbono en el estado sólido. Finalmente, esto da menor penetración a los átomos de carbono, dando lugar a que el contenido de carbono sea menor que en los procesos con reductor gaseoso, del orden de 0,1-0,2 \%.

La mayoría de procesos que operan a base de carbón utilizan hornos de tipo rotatorio. En este tipo de hornos se produce segregación de la carga durante la rotación debido a diferencias de densidad y tamaño de los materiales de carga y a la inclinación del mismo. La separación del hierro metálico, al final del proceso, es posible, debido a que el resto de la carga no es magnética. En los hornos rotatorios el material se reduce en un horno y el enfriamiento se realiza en otro, con objeto de controlar ambas etapas. En la zona de salida del horno de reducción, la cantidad de carbón activo se reduce, por lo que en esa zona, generalmente, se inyectan finos de carbón.

El hierro esponja producido en hornos rotatorios tiene baja tendencia a la reoxidación, debido a que durante el movimiento rotativo muchos de los poros se cierran, además de que las temperaturas de reducción son más altas que en los procesos con reductor gaseoso.

El mejor tipo de carbón no coquizable a utilizar en los hornos rotatorios debe tener un contenido no muy elevado de cenizas ya que éstas reducen el volumen disponible del horno. Se prefieren contenidos máximos de ceniza de $24 \%$. Así mismo, la temperatura de fusión de la ceniza debe ser 
alta para evitar la formación de costras. La reactividad del carbón debe ser alta, preferentemente. Esta característica se mide por la tendencia del carbono para reaccionar con $\mathrm{CO}_{2}\left(\mathrm{~cm}^{3} \mathrm{CO} / \mathrm{g} \mathrm{C}\right)$. Una ventaja adicional de trabajar con carbón de alta reactividad consiste en que la diferencia de temperaturas entre la atmósfera del horno y la carga disminuye, indicando una mejor transferencia de calor entre ambas fases y reduciendo, a la vez, la posibilidad de puntos calientes, los cuales promueven la formación de costras que se adhieren al horno. En general la diferencia de temperatura entre la carga y el gas es del orden de 100 . $150^{\circ} \mathrm{C}$.

El llenado de un horno rotatorio varia de 12 a $15 \%$. Con este volumen de llenado se asegura un perfil de temperatura adecuado para lograr la temperatura de reducción. Chatterjee ${ }^{[7]}$ ha encontrado que, para lograr más del $95 \%$ de la reducción, se debe precalentar la carga hasta $600{ }^{\circ} \mathrm{C}$ en el $15 \%$ de la longitud total del horno. La temperatura del horno se controla mediante sensores, los cuales inyectan la cantidad de aire necesario para mantener la temperatura deseada. El avance de la carga depende de la velocidad de rotación y del ángulo de inclinación del horno.

Los procesos a base de carbón con horno rotatorio tienen características similares. Las variantes principales están relacionadas con sistemas para controlar el perfil de temperatura del horno.

El otro tipo de horno empleado en procesos base carbón es el horno de hogar giratorio (HHG). En este horno, el mineral de hierro no esta sujeto a esfuerzos mecánicos importantes, de manera que el pelet se puede emplear sin la etapa de endurecimiento. La carga esta compuesta generalmente por pelet compuesto, el cual se forma con una mezcla de finos de mineral y finos de carbón, o bien es posible cargar directamente finos de mineral y carbón al horno.

\subsection{1. $S L / R N$}

Los datos, hasta 1999, indican que este proceso es el mas empleado del grupo de procesos que emplean carbón no coquizable como agente reductor. El horno es un típico horno rotatorio. El horno tiene dos zonas importantes, una de precalentamiento (900-1.100 ${ }^{\circ} \mathrm{C}$ ) y otra de reducción, en la cual la temperatura permanece uniforme (1.050-1.100 $\left.{ }^{\circ} \mathrm{C}\right)$. El DRI se descarga del horno, a una temperatura de $1.000^{\circ} \mathrm{C}$, a un enfriador rotatorio. El enfriamiento se realiza de manera indirecta con agua rociada sobre la superficie del cilindro rotatorio. Los gases de salida se someten a postcombustión. En las versiones actuales de este proceso (Tabla X) se cuenta con toberas de aire sumergidas para mejorar el calentamiento, realizando la postcombustión de una fracción de los gases generados.

\subsection{2. $C O D I R$}

El proceso CODIR (Coal-Ore-Direct-Reduction) es muy similar al proceso SL/RN. En este proceso (Tabla XI) se enriquece el aire de soplo con oxígeno $\left(23 \% \mathrm{O}_{2}\right)$, con lo cual se obtiene un consumo de energía menor. Adicionalmente, se introduce carbón, con un déficit de aire en la zona de salida del reactor de reducción para controlar la temperatura.

\subsubsection{ACCAR}

El proceso ACCAR (Allis-Chalmers-ControlledAtmospheric-Reduction) es similar a los anteriores. Su característica principal es la inyección de combustible para calentar el carbón cuando la carga esta sobre las toberas, con lo que, adicionalmente, se produce también gas reductor. Con este combustible complementario se logran cantidades elevadas de carbono en el producto, similares a las alcanzadas en los de procesos con reductor gaseoso. El consumo de energía es similar al proceso CODIR. La única planta en operación se encuentra en la India. El proceso se comercializa ahora por la empresa Orissa Sponge Iron Ltd., por lo cual el proceso se ha renombrado como OSI L.

Tabla X. Características del proceso SL/RN

Table $X$. Features of $S L / R N$ process

\begin{tabular}{ll}
\hline & SL/RN \\
\hline Inicio & 1964 \\
Desarrollado por & Stelco-Lurgi-Republic Steel- \\
& National Lead Co., Canada \\
Tipo de horno & Rotatorio \\
Reductor & Carbón \\
Temp. reducción & $1.000-1.100^{\circ} \mathrm{C}$ \\
Carga & Mineral o pelet, carbón y caliza \\
Energía & $19,9 \mathrm{GJ} / \mathrm{t}$ \\
\hline
\end{tabular}


Tabla XI. Características del proceso CODIR

Table XI. Features of CODIR process

\begin{tabular}{ll}
\hline & CODIR \\
\hline Inicio & 1974 \\
Desarrollado por & Krupp; Alemania \\
Tipo de horno & Rotatorio \\
Reductor & Carbón \\
Temp. reducción & $1.000-1.100^{\circ} \mathrm{C}$ \\
Carga & Mineral o pelet, carbón y caliza \\
Consumo carbón & $380-400 \mathrm{~kg} / \mathrm{t} \mathrm{DRI}$ \\
Energía & $15,0 \mathrm{GJ} / \mathrm{t}$ \\
\hline
\end{tabular}

\subsection{4. $D R C$}

El proceso DRC (Davy Direct Reduction Corporation) resultó de una evolución del proceso HOCKINS (Australia). Se inyecta aire en todo el horno, con inyección de finos de carbón por la zona de salida. Es el segundo proceso comercial más importante.

\subsubsection{KINGLOR METOR}

Proceso desarrollado por Danieli en 1971. El reactor es un horno vertical. El calor se obtiene mediante combustión de gas, transmitiéndose a la carga a través de unas paredes que son de carburo de silicio. La carga esta compuesta por mineral de hierro y carbón.

\subsection{6. $T D R$}

El proceso TDR, desarrollado por la empresa India Tata Steel cuenta con inyección de aire a lo largo del horno, con toberas axiales y radiales.

\subsubsection{COMET}

El COMET(COal based METallization) es un proceso desarrollado por el Centro de Investigaciones Metalúrgicas (C.R.M.) de Bélgica ${ }^{[8]}$ que emplea un horno de hogar rotatorio para reducir óxidos de hierro en forma de finos. Su característica principal es la producción de DRI de bajo contenido de azufre $(0,03-0,07 \%)$. En este proceso se alternan capas de finos de mineral de hierro con capas de finos de carbón y caliza. En la zona de reducción se alcanzan temperaturas del orden de $1.300^{\circ} \mathrm{C}$, con lo cual se logran altas metalizaciones y cortos tiempos de proceso. El número óptimo de capas de mineral de hierro varia de 2 a 3, con un espesor que puede variar de 1 a $20 \mathrm{~mm}$. Este proceso presentó problemas relacionados con una deficiente transferencia de calor debido a la cantidad total de capas.

\subsubsection{INMETCO}

Proceso comercializado por Mannesmann-Demag. El proceso INMETCO emplea carbón no coquizable, finos de mineral y caliza, en un horno de hogar giratorio. En su esquema tradicional, los finos de mineral se aglomeran con finos de caliza y carbón. El pelet se carga al horno sin someterse a secado, debido a que no esta sujeto a grandes esfuerzos mecánicos dentro del horno y se carga en forma de capas uniformes. En el producto se reportan concentraciones de carbono hasta de $5 \%$. Este proceso (Tabla XII) se desarrolló, inicialmente, para reciclar desechos de plantas metalúrgicas, siendo en la actualidad uno de los procesos más importantes para el reciclado de desechos ${ }^{[9]}$.

Los principales desechos de plantas metalúrgicas son diversos polvos y la cascarilla de laminación. Los polvos están constituidos por partículas muy pequeñas, generalmente menores a $1 \mu$ y su transporte, por medios neumáticos, es simple. La cascarilla puede contener aceite, lo que dificulta su recuperación, requiriendo una limpieza previa. El polvo, en acerías que funden chatarra tiene, generalmente, elevados contenidos de zinc, que junto con el plomo se elimina parcialmente a través de los gases de salida durante la reducción del mineral.

\subsubsection{FASTMET}

El proceso FASTMET es la versión MIDREX para procesos con reductor sólido. Es muy similar al

Tabla XII. Características del proceso INMETCO

Table XII. Features of INMETCO's process

\begin{tabular}{ll}
\hline & INMETCO \\
\hline Inicio & 1964 \\
Desarrollado por & INCO, Canada \\
Tipo de horno & Hogar giratorio \\
Reductor & Carbón \\
Temp. reducción & $1.250-1.350^{\circ} \mathrm{C}$ \\
Carga & Finos de mineral, carbón y caliza \\
Consumo carbón & $700 \mathrm{~kg} / \mathrm{t} \mathrm{DRI}$ \\
Energía & $16 \mathrm{GJ} / \mathrm{t}$ \\
\hline
\end{tabular}

http://revistademetalurgia.revistas.csic 4287 
proceso INMETCO, con la diferencia de que, en este proceso, los pellets compuestos se secan antes de ser introducidos al reactor de hogar rotatorio. En su esquema, el producto se carga en caliente al horno eléctrico de arco. Fue el primer proceso empleando horno giratorio para producir DRI. En el proceso se coloca una sola capa de mineral, operando a altas temperaturas de reducción, $1.340^{\circ} \mathrm{C}$, logrando una alta transferencia de calor.

\subsubsection{DRYIRON}

Proceso similar a FASTMET, desarrollado por Maumme Research de EE. UU. ${ }^{[10]}$. Se caracteriza por emplear un exceso de carbón para producir una alta concentración de gas reductor. La concentración de carbono en el producto es alta, del orden de $4 \%$.

\subsubsection{HI-QIP}

El proceso HI-QIP (HIgh Quality Iron Pebble) es reciente $^{[11]}$, ha sido desarrollado por la empresa japonesa Kawasaki e introduce un concepto tecnológico sobresaliente: la producción de un material sólido, libre de escoria, en un solo horno. El diseño del horno consiste en una banda rotatoria que contiene perforaciones en las que se deposita el material fundido durante la última fase de reducción. La primera capa es de carbón, con un espesor de $30 \mathrm{~mm}$, sobre la cual se coloca una capa de, aproximadamente $15 \mathrm{~mm}$, conteniendo la mezcla de finos de mineral y cal. Al efectuarse la fusión del mineral, la escoria se separa. El producto permanece por encima de la capa de carbón debido a fuerzas tensoactivas. Sus creadores señalan que aumentando el espesor de la capa de mineral de hierro disminuye la productividad.

\section{PROCESOS DE REDUCCIÓN EN ESTADO LÍQUIDO (SMELTING)}

\subsection{Procesos de fusión reductora}

Los procesos de "smelting" o fusión reductora representan alternativas al horno alto para producir hierro líquido. Este término indica que la reducción del óxido de hierro se efectúa, aun parcialmente, en estado líquido. En general, en estos procesos, la reducción en estado sólido se efectúa en un reactor y la fusión-reducción en otro y se emplea carbón no coquizable. En estos procesos se realizan las mismas reacciones que en el horno alto, pero de una manera más controlada y acelerada. El mineral de hierro se reduce parcialmente, generalmente a $\mathrm{FeO}$, en el reactor de reducción y se transfiere por gravedad al reactor de fusión, donde el óxido de hierro entra en contacto con la escoria, siendo reducido por partículas de carbón o por carbono disuelto en el metal. En una sección intermedia entre ambos reactores se efectúan las reacciones de gasificación del carbono. Las reacciones (4) y (5) representan las reacciones de reducción del óxido de hierro en estado líquido mediante finos de carbón y el carbono disuelto en el metal, respectivamente y, las reacciones (6) y (7), las reacciones de gasificación:

$$
\begin{aligned}
& \mathrm{FeO}_{(\mathrm{l})}+\mathrm{C}_{(\mathrm{s})}=\mathrm{Fe}_{(\mathrm{l})}+\mathrm{CO}_{(\mathrm{g})} \\
& \mathrm{FeO}_{(\mathrm{l})}+\underline{\mathrm{C}}=\mathrm{Fe}_{(\mathrm{l})}+\mathrm{CO}_{(\mathrm{g})} \\
& \mathrm{C}_{(\mathrm{s})}+\mathrm{CO}_{2(\mathrm{~g})}=2 \mathrm{CO}_{(\mathrm{g})} \\
& \mathrm{H}_{2} \mathrm{O}_{(\mathrm{g})}+\mathrm{C}_{(\mathrm{s})}=\mathrm{H}_{2(\mathrm{~g})}+\mathrm{CO}_{(\mathrm{g})}
\end{aligned}
$$

A diferencia del horno alto, en los procesos de fusión reductora se generan grandes temperaturas en la superficie del metal líquido debido a la postcombustión del CO:

$$
\mathrm{CO}_{(\mathrm{g})}+1 / 2 \mathrm{O}_{2(\mathrm{~g})}=\mathrm{CO}_{2(\mathrm{~g})}
$$

Cuando se emplea aire para la reacción de postcombustión, la temperatura se incrementa debido a que el nitrógeno diluye la influencia del $\mathrm{H}_{2} \mathrm{O}$ y $\mathrm{CO}_{2}$ en reacciones inversas. El aumento de temperatura del baño metálico se ve limitado por la acción enfriante de la reacción de Boudouard y la transferencia de calor a través de una alta columna de escoria espumosa. Además de la baja transferencia de calor al baño metálico, el alto valor del calor sensible del gas de salida también contribuye a proporcionar una baja eficiencia térmica a los procesos de fusión reductora.

Los primeros trabajos sobre fusión reductora los condujeron Wiberg (1938) y Engell (1939). Sin embargo, no atrajeron mucha atención debido al gran interés que despertaron los procesos de reducción directa a fines de los años cincuenta.

\subsubsection{COREX}

El proceso COREX es, de los procesos de fusión reductora, el único que ha logrado alcanzar la etapa comercial empleando carbón no coquizable. Este 
material se carga en la parte superior del reactor de fusión, donde se gasifica. El oxígeno que se introduce para quemar el carbón (tamaño, 10-40 $\mathrm{mm}$ ) se inyecta a una velocidad tal, que logra fluidizarlo. El gas que sale del reactor de fusión, compuesto por un $95 \%\left(\mathrm{H}_{2}+\mathrm{CO}\right)$, con un alto poder reductor, se combina con una porción del gas reciclado proveniente del reactor de reducción, con lo cual se enfría a una temperatura de 800-900 ${ }^{\circ} \mathrm{C}$ y, después de eliminar el polvo en un precipitador, se introduce al reactor de reducción. El gas de salida se recicla, después de eliminar el $\mathrm{CO}_{2}$ (Tabla XIII). Este gas tiene un alto poder calorífico, $7.500 \mathrm{~kJ} / \mathrm{Nm}^{3}$, equivalente al $50 \%$ de la energía contenida en todo el carbón empleado en el proceso $^{[12]}$, de manera que debe aprovecharse, necesariamente, con eficacia, para que el proceso sea rentable.

El proceso COREX se considera más versátil que el horno alto debido a que puede pararse más fácilmente cuando no se requiere arrabio ${ }^{[13]}$. La primera planta comercial se instaló en ISCOR (Sudáfrica) en 1989, con una capacidad de 0,4 mtpa y en 1995 se instaló una segunda planta de mayor capacidad (0,8 mtpa) en POSCO (Corea del Sur) y, más recientemente, en 1999, en otras tres empresas: HANBO (Corea del Sur), JINDAL (India) e ISCOR-SALDANHA (Sudáfrica).

Tabla XIII. Características del proceso COREX

Table XIII. Features of COREX process

\begin{tabular}{ll}
\hline & COREX \\
\hline Inicio & 1981 \\
Desarrollado por & VAl-KORF Eng; Alemania \\
Tipo de horno & Vertical \\
Presión & Hasta 5 atm \\
Reductor & Gasificación de C \\
Composición & $72 \%$ CO-12 $\% \mathrm{H}_{2}-12 \% \mathrm{CO}_{2}$ \\
Temp. reducción & $850{ }^{\circ} \mathrm{C}$ \\
Temp. postcombustión & $1.500-1.800^{\circ} \mathrm{C}$ \\
Temp. gasificador & $1.000-1.300^{\circ} \mathrm{C}$ \\
Carga & Pelet \\
Consumo carbón & $600 \mathrm{~kg} / \mathrm{t} \mathrm{DRI}$ \\
Consumo O & $500-700 \mathrm{Nm}{ }^{3} / \mathrm{t} \mathrm{DRI}$ \\
Energía & $15,5 \mathrm{GJ} / \mathrm{t}$ \\
\hline
\end{tabular}

\subsubsection{AISI}

El proceso AISI es similar a COREX. Emplea dos reactores. Se sopla oxígeno por la parte superior e inferior del reactor de fusión reductora, con lo cual el grado de oxidación final de la escoria es mayor que en el proceso COREX y, en consecuencia, resulta un metal con un bajo contenido en silicio y con mayor contenido de azufre.

\subsubsection{FINEX}

El proceso FINEX ${ }^{[14]}$ se desarrolló por el mismo técnologo que desarrollo el proceso COREX. La diferencia de COREX y FINEX consiste en que, en este ultimo, se emplea un esquema de reducción como el que emplea el proceso FIOR, de fluidización, con varios reactores para prereducir finos de mineral de hierro. Posteriormente, estos finos son cargados a un reactor de fusión reductora para su reducción final.

\subsubsection{HISMELT}

Este proceso se caracteriza por una agitación muy intensa, debido a la inyección directa, al baño líquido, de finos de mineral, previamente reducidos, y de carbón. En su primera versión (1991), la planta piloto constaba de un horno horizontal. Posteriormente, en 1997, este horno se modificó a horno vertical. Sus creadores señalan ${ }^{[15 \text { y } 16]}$ que, con esta modificación se eliminaron problemas de obstrucción en las boquillas de inyección sumergidas por el fondo y una reducción importante en el consumo de refractario (de 25 a $2 \mathrm{~kg} / \mathrm{t}$ arrabio). En la nueva versión se inyecta, adicionalmente, un flujo de aire enriquecido con oxígeno (20-30\%), por la parte superior, el cual se emplea para la postcombustión de los gases de salida. En este esquema, la escoria resultante, ( $5 \% \mathrm{FeO})$, es mucho más oxidante que la del horno alto $(0,5-1$ $\% \mathrm{FeO}$ ), lo cual presenta ventajas para la defosforación. Los niveles de postcombustión son altos, del orden del $60 \%$. Con estos niveles de postcombustión, es necesario introducir algún hidrocarburo al gas de salida del horno de fusión para incrementar su poder reductor. La inyección de finos de carbón, al baño metálico, asegura una mayor recuperación de carbono y genera una gran turbulencia, con lo que aumenta la velocidad de las reacciones químicas. Se señala, adicionalmente, que las reacciones de reducción se efectúan directamente dentro del metal, a diferencia de otros 
procesos de fusión reductora en los cuales se efectúa en la escoria espumosa.

\subsubsection{DIOS}

El proceso DIOS (Direct Iron Ore Smelting) es un proceso desarrollado por la Federación Japonesa del Hierro y el Acero (JISF) y, al igual que el proceso HISMELT, también emplea un horno de lecho fluidizado para prereducir finos de mineral de hierro y un horno de fusión (SRF). La planta piloto inició operaciones en 1993. A diferencia del proceso HISMELT, en este proceso, la lanza vertical inyecta oxígeno puro para efectuar las reacciones de combustión y postombustión y los finos de carbón y mineral se inyectan por la parte superior del horno de fusión reductora. Los finos de carbón, en su descenso, reaccionan con el oxígeno generando calor. Los finos de mineral inyectados se reducen en dos hornos, logrando niveles de prereducción del orden de $25 \%$. La diferencia principal de este proceso con el proceso AISI consiste en que, en este último, se emplea un reactor de reducción vertical convencional, logrando niveles de prereducción mayores, del orden del $30 \%$, mientras que el proceso DIOS emplea hornos de lecho fluidizado.

\subsubsection{REDSMELT}

Este proceso está compuesto por dos unidades: un horno de hogar rotatorio, donde se efectúa la reducción, y un horno de fusión. El mineral, en forma de pellets sin cocer $(6-12 \mathrm{~mm})$, se coloca en 1-2 capas dentro del horno rotatorio, a una temperatura de $1.370{ }^{\circ} \mathrm{C}$. El horno de fusión es un horno eléctrico del tipo empleado en la producción de ferroaleaciones. Este proceso es desarrollado por la empresa alemana Mannesmann-Demag. Sus creadores proponen ${ }^{[17]}$ el empleo de arrabio líquido, directamente en hornos eléctricos, para producir acero.

\subsubsection{FASTMELT}

Es un proceso muy similar a REDSMELT, desarrollado por MIDREX-Kobe, al cual incorporan su proceso FASTMET para reducir mineral de hierro con carbón, cargando, posteriormente, el material prereducido a un horno eléctrico de coraza fija ${ }^{[18]}$. Los costos de inversión de una planta FASMET, con capacidad para producir $0,5 \mathrm{mtpa}$ se estiman en 90-100 millones de dólares y, para una planta FASTMELT, en 115-125 millones de dólares ${ }^{[19]}$.

\subsubsection{ROMELT}

El proceso ROMELT no emplea horno para prereducir el mineral de hierro, siendo uno de los procesos, en etapa de desarrollo, más nuevos. El mineral y el resto de la carga se introducen directamente en el horno de fusión reductora, donde se introduce aire enriquecido con oxígeno, mediante toberas. Se señala ${ }^{[20]}$ que este proceso presenta la desventaja de un alto consumo de energía, debido a carecer de la etapa de prereducción y, también, un alto consumo de agua de enfriamiento del horno.

\subsubsection{TECNORED}

El proceso TECNORED es otro proceso que no emplea la etapa de pre-reducción del mineral de hierro. El horno de fusión reductora tiene la forma de horno de cubilote. El mineral de hierro se reduce en la parte central del horno y se funde en el etalaje. El horno opera a 1,5 atm.

Existen varios procesos que emplean energía eléctrica para realizar la fusión de la carga en el horno de fusión reductora, tales como ELRED, INRED, COMBISMELT Y PLASMASMELT. En estos procesos, la energía eléctrica puede generarse produciendo vapor con los gases y con distintos resultados, en cuanto al balance de energía eléctrica.

\subsubsection{MINI- HORNO ALTO (MBF)}

El mini-horno alto es una adecuación de los grandes hornos altos a condiciones de producción de arrabio en menor escala (Tabla XIV). Se clasifican en tres tipos: los hornos altos, cuyo volumen de trabajo es superior a $2.000 \mathrm{~m}^{3}$; medianos, de $500 \mathrm{a}$ $1.500 \mathrm{~m}^{3}$; y, pequeños, si el volumen de trabajo es menor a $500 \mathrm{~m}^{3}$. El tamaño de los hornos altos varia de 1.500 a $5.000 \mathrm{~m}^{3}$. El valor estándar

Tabla XIV. Características del mini horno alto

Table XIV. Features of mini blast furnaces

\begin{tabular}{ll}
\hline \multicolumn{2}{c}{ MINI-HORNO ALTO } \\
\hline Volumen trabajo & $200-400 \mathrm{~m}^{3}$ \\
Reductor & coque-carbón \\
Carga & sinter \\
Consumo carbón vegetal-coque & $550 \mathrm{~kg} / \mathrm{t} \mathrm{DRI}$ \\
Presión & $0,3 \mathrm{~kg} / \mathrm{cm}^{2}$ \\
Productividad & $2,5-3 \mathrm{t} / \mathrm{m}^{3} \mathrm{~d}$ \\
\hline
\end{tabular}


del volumen de trabajo se refiere al volumen entre la línea de toberas y la línea de carga; sin embargo, en China incluyen un volumen de trabajo mayor, hasta la línea del orificio de vaciado. China, además de ser el país que produce más arrabio a nivel mundial es, también, el que tiene la mayor cantidad de mini-hornos altos (3.100 MBF), en los que se obtiene alrededor del $64 \%$ de su producción nacional.

Se identifican las siguientes ventajas ${ }^{[21}$ y 22$]$ de los MBF, con respecto a los hornos altos convencionales: menor inversión inicial, menores costos de operación y mayor flexibilidad. Sin embargo, la productividad no es mayor debido a que el consumo de combustible es alto, lo cual es consecuencia de mayores perdidas de calor (17\% para $\mathrm{MBF}$ contra $5-10 \%$ para $\mathrm{BF}$ ), lo que obliga a emplear mayor cantidad de carbón. Además, debido a la baja altura de los MBF, la eficiencia de utilización del gas reductor es menor. Por otra parte, operativamente, el proceso se comporta de manera similar, obteniendo una mayor productividad al aumentar la calidad del mineral (los minerales de hierro chinos son de muy baja calidad, en promedio, del $33 \% \mathrm{Fe}_{\mathrm{t}}$ ), la temperatura del viento (en el rango de 800 a $1.000^{\circ} \mathrm{C}$ ) y la proporción de sinter en la carga metálica.

Los niveles de productividad que se mencionan en la tabla anterior son los que se han logrado utilizando coque. Se señala el carbón vegetal como reductor, debido a que este ha sido el reductor planeado originalmente.

\section{EVALUACION DE PROCESOS}

\subsection{Procesos de reducción directa}

A principios de los años sesenta, se estimaba que los procesos de reducción directa dominarían la producción de hierro. 40 años después, estos procesos no han logrado realmente ese objetivo. Las causas de este hecho pueden ser las siguientes limitaciones generales de los procesos de RD:

- Baja productividad.

- Problemas de manejo del producto.

- Altos costos de producción.

- El DRI produce más escoria durante su fusión, consumiendo mayor energía y fundentes.

La tabla XV muestra las ventajas y desventajas de los diversos procesos para producir DRI. La ventaja principal de los procesos de reducción directa base-carbón es el uso de una fuente de energía barata y abundante. Los procesos que emplean hornos de hogar rotatorio presentan la ventaja adicional de que, en general, pueden emplear directamente finos de mineral de hierro sin la etapa de aglomeración. Frente a estas ventajas los procesos tienen una serie de desventajas, tales como un alto consumo de energía, obtención de un producto mezclado con cenizas, etc. Los procesos base-gas, por el contrario, emplean una fuente de energía costosa y, en general, requieren de la etapa de

Tabla XV. Comparación de procesos de reducción directa

Table XV. Comparison of direct reduction processes

Procesos de reducción directa

\begin{tabular}{|c|c|c|}
\hline & Base carbón & Base Gas \\
\hline Ventajas & - Utiliza una fuente de energía barata: el carbón & $\begin{array}{l}\text { - } \text { Mayor facilidad de control } \\
\text { - } \text { Mayor disponibilidad del volumen } \\
\text { de trabajo del reactor } \\
\text { - } \quad \text { Mayor } \% \text { C y menor } \% \mathrm{~S} \text { en el producto } \\
\text { - } \quad \text { Producto más limpio } \\
\text { - } \quad \text { Mayor productividad }\left(2 \mathrm{t} / \mathrm{m}^{3} \mathrm{~d}\right) \\
\text { - }\end{array}$ \\
\hline Desventajas & $\begin{array}{l}\text { - Tamaño máximo de planta de } 0,2 \text { mtpa. Los costos } \\
\text { de inversión limitan plantas más grandes }{ }^{[26} \text { y 27] } \\
\text { - El producto contiene de } 1-2 \% \text { de ceniza } \\
\text { - Mayor } \% \mathrm{~S}(0,020-0,025) \text { y menor } \% \mathrm{C}(0,2 \%) \\
\text { - Menor productividad }\left(0,3-0.5 \mathrm{t} / \mathrm{m}^{3} \mathrm{~d}\right)^{[5]} \\
\text { - Mayor consumo de energía }(16-21 \mathrm{GJ} / \mathrm{t} \mathrm{DRI}) \\
\text { - Producen mayor cantidad de escoria requiriendo } \\
\text { mayor cantidad de fundentes y energía durante su fusión }\end{array}$ & $\begin{array}{l}\text { - Muy alta dependencia del gas natural } \\
\text { - } \quad \text { Producto pirofórico }\end{array}$ \\
\hline
\end{tabular}


aglomeración; no obstante, su predominio es evidente, con casi un 93 \% de la producción mundial, gracias a producir un material más limpio y de manera más controlada.

La decisión para seleccionar el mejor proceso estará influenciada, finalmente, por sus costos de producción y por su flexibilidad. Lüngen y Steffen ${ }^{[23]}$ realizaron un estudio comparativo de costos entre algunos procesos alternativos al horno alto. Los factores que consideraron fueron el costo de inversión y los factores de consumo. Los autores informan de un precio del gas natural en Alemania 7,7 veces superior al precio del gas natural en Venezuela. Esta es la razón principal por la cual, hasta ahora, solamente los países con posibilidades de gas natural a buen precio son los que producen mas hierro esponja. En su análisis y para las condiciones de Alemania, señalan que los procesos con costos de inversión más elevados son el horno alto (300 US $\$ / t$ ) mientras que, empleando el proceso MIDREX, el costo se ubica en 180 US $\$ / t$. Sin embargo, después de evaluar factores de operación, concluyen que los costos de producción menores son para el proceso de horno alto (145 US\$/t), seguido de los procesos COREX (160 US $\$ / t$ ) y MIDREX (165 US\$/t).

MIDREX $^{[24]}$ señala un costo de producción de $140 \mathrm{US} \$ / \mathrm{t}$ para condiciones alemanas, mientras que para condiciones de Venezuela los costos son mucho menores, 90-110 US\$/t. Para la venta de DRI a Europa estiman un precio de 150-170 US $\$ / t$.

La gran dependencia del gas natural en los procesos de reducción directa base-gas es uno de sus aspectos más críticos. Durante la segunda mitad del año 2000, los precios del gas natural en Norteamérica se incrementaron drásticamente, afectando particularmente a México, el país productor más importante de hierro esponja a nivel mundial, con una producción de $16 \%$ en 1999. El aumento del precio del gas natural fue de 2,37 US\$/GJ (2,5 US $\$ / M B t u)$ a 9,47 US $\$ / G J$ (10 US $\$ / M B t u)$. Este dramático aumento del precio del gas natural afectará la producción de hierro esponja en los próxi-

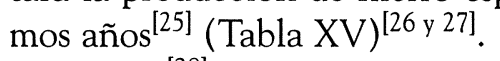

Whipp ${ }^{[28]}$ realizó una comparación sobre factores de consumo de los procesos de reducción directa, la cual se muestra en la tabla XVI.

Este análisis concluye que los costos de producción son menores cuando se emplean procesos de reducción directa que utilizan finos de mineral de hierro, en particular los procesos IC y FINMET. Sin embargo, se parte de estimaciones sobre proce-
Tabla XVI. Factores de consumo de procesos de reducción directa

Table XVI. Consumption factors of DRI processes

\begin{tabular}{lcccc}
\hline Proceso & $\begin{array}{c}\text { t mineral/ } \\
\text { t DRI }\end{array}$ & $\begin{array}{c}\text { kwh/ } \\
\text { t DRI }\end{array}$ & $\begin{array}{c}\text { MMBtu/ } \\
\text { t DRI }\end{array}$ & $\begin{array}{c}\text { Kg C/ } \\
\text { t DRI }\end{array}$ \\
\hline $\begin{array}{l}\text { Hornos verticales } \\
\text { Hornos de lecho }\end{array}$ & $1,43-1,45$ & $110-130$ & 10 & \\
$\begin{array}{l}\text { fluidizado } \\
\text { Hornos de hogar }\end{array}$ & $1,47-1,48$ & $110-150$ & $11-12$ & \\
giratorio & & & & \\
Hornos Rotatorios & $1,43-1,40$ & $90-120$ & $2-3$ & $0,3-0,4$ \\
Carburo de hierro & 1,39 & $70-100$ & 0 & 0,75 \\
\hline
\end{tabular}

sos que cuentan con una experiencia de producción muy limitada. Con relación al lugar de producción es evidente que, en regiones donde el precio del gas natural es menor, será posible reducir los costos de producción.

Los procesos HYL y MIDREX parecen haber alcanzado niveles de madurez similares en la actualidad. Quizás, una posible explicación acerca de porqué MIDREX supera en volumen de producción al proceso HYL, sea la de haberse adelantado en sus avances tecnológicos, cómo son la introducción de horno vertical, auto-reformación y otros y de que, en consecuencia, sus estrategias de control pueden estar más afinadas.

\subsection{Procesos de fusión reductora}

Una ventaja de los procesos de fusión reductora sobre los procesos de reducción directa consiste en que la calidad del mineral de hierro empleado en procesos de fusión reductora puede ser menor que para procesos de reducción directa, debido a que durante la fusión se separa la escoria. Por otra parte, Chatterjee ${ }^{[29]}$ menciona que una de las principales desventajas de los procesos de fusión reductora es el alto costo de inversión de las instalaciones auxiliares, tales como la planta de oxígeno y la planta de generación de energía eléctrica, que representan más del $50 \%$ del costo de la inversión y que, para que estos procesos sean competitivos con respecto al horno alto, se debe emplear óptimamente el gas de salida, ya sea para generar energía eléctrica o para otros usos.

Por otra parte, en los procesos de fusión reductora se obtiene, generalmente, un producto que tiene una mayor concentración de azufre que la del el arrabio de horno alto debido al uso directo de carbón, el cual contiene más azufre que el 
coque y, a que la escoria es de naturaleza menos reductora. La ventaja aparente de no aglomerar el concentrado de mineral y ahorrarse la etapa de peletización en algunos procesos, como TECNO. RED, se señala, a la vez, como desventaja ${ }^{[30]}$ debido a que requieren un tamaño muy fino $(<250 \mu)$, lo cual exige etapas adicionales para pulverizar el mineral.

A pesar de que el proceso COREX es el único de su tipo que ha alcanzado la escala comercial, presenta algunas limitaciones, debido a que no emplea finos de mineral por lo que, por una parte, requiere aglomeración y, por otra parte, la reducción en su primera fase es más lenta que si se redujesen finos de mineral, Adicionalmente, su talón de Aquiles es el alto contenido calorífico del gas de salida, el cual debe, necesariamente, aprovecharse para producir energía o como agente reductor en otro proceso y, así, lograr un bajo consumo de energía. Se ha logrado acoplar el gas de salida con el proceso MIDREX, pero esto exige una sincronización de operaciones muy rígida.

La tabla XVII compara los valores de composición del metal obtenido en el horno alto con otros procesos de fusión reductora.

Los procesos de fusión reductora han demostrado la importancia de separar las diferentes etapas que se suceden en el horno alto con objeto de controlar cada una de ellas de manera más eficiente y, en general, emplean carbón no coquizable, lo que contribuye a aliviar la gran presión sobre el control ambiental en las empresas siderúrgicas. Sin embargo, falta aun mucho más trabajo de investigación para optimizar las variables de proceso. Fruehan ${ }^{[31]}$ señala los siguientes aspectos a optimizar:

- La postcombustión de $\mathrm{H}_{2}$ y $\mathrm{CO}$ a vapor de agua y $\mathrm{CO}_{2}$, respectivamente, que debe ser mayor al $40 \%$ para mejorar la transferencia de calor.

Tabla XVII. Composición química del producto en procesos de smelting

Table XVII. Chemistry of metal and slag in smelting processes

\begin{tabular}{lccccc}
\hline & \multicolumn{3}{c}{ Metal, (\% masa) } & \multicolumn{2}{c}{ Escoria, (\% masa) } \\
\hline & $\mathrm{C}$ & $\mathrm{S}$ & $\mathrm{Si}$ & $\mathrm{CaO} / \mathrm{SiO}_{2}$ & $\mathrm{FeO}$ \\
\hline Horno Alto & $4-5$ & 0,05 & $0,40-0,50$ & $1-1,3$ & $0,5-1$ \\
AISI, & & & & & \\
HISMELT & $4-5$ & $0,15-0,20$ & - & $1-1,3$ & $2-5$ \\
$\begin{array}{l}\text { DIOS } \\
\text { COREX }\end{array}$ & $4-5$ & $0,05-0,10$ & $0,40-0,80$ & $1-1,3$ & $1-2$ \\
TECNORED & & & & & \\
\hline
\end{tabular}

- La mejora de la prerreducción, empleando finos de mineral en lechos fluidizados.

- El diseño de métodos eficientes de desulfuración del metal líquido. El metal de un proceso de fusión reductora tiene hasta cuatro veces más azufre que el arrabio producido en el horno alto.

El proceso empleando mini-horno alto ha tenido un auge importante en China, aprovechando sus ventajas de menor costo de inversión y mayor flexibilidad de operación. Sin embargo, debido a sus limitaciones relacionadas con un alto consumo de energía, una eficiencia menor de utilización del gas reductor que la del horno alto y la continuidad en la dependencia del coque para lograr buenos índices de productividad, no se le puede considerar como un proceso sustitutivo.

\section{CONCLUSIÓN}

No se advierte que ningún proceso alternativo compita en el medio plazo con el horno alto para lograr sus niveles de producción, utilizando como plataforma de operación sus desventajas, tales como, altos costos de inversión, poca flexibilidad y alta dependencia del coque metalúrgico, manteniéndose aun como un gran reto tecnológico. Sin embargo, la producción de arrabio, muy probablemente continuará prácticamente estancada, no sucediendo lo mismo con la producción de prerreducidos, que tendrán una demanda creciente para el horno eléctrico de arco, con objeto de diluir los contenidos de elementos residuales de la chatarra.

La contribución tecnológica de los procesos de fusión reductora ha sido la de aplicar un control más estrecho e intenso de cada una de las etapas de la reducción del mineral de hierro, eliminar su etapa de aglomeración y el empleo de carbón vegetal. Sus esquemas de proceso consideran "ciclos cerrados" de reutilización de la energía y por ello presentan un gran potencial futuro.

La competencia entre los procesos HYL y MIDREX continuara. Sin embargo, los resultados dependerán más de sus estrategias de control para asegurar homogeneidad y consistencia del producto que de las diferencias entre ambos procesos. Por otra parte, su mayor reto seguirá siendo el aumento de productividad en base a un aumento de la velocidad de reducción y al grado de utilización del gas reductor.

Los procesos de reducción directa base-gas que emplean finos de mineral de hierro no han logrado 
desplazar a los procesos que emplean pelet, debido a problemas de fluidización y mayor tiempo de reducción. Sin embargo, esta ruta es interesante ya que se elimina la etapa de aglomeración.

\section{REFERENCIAS}

[1] IISI forecast, 1999. www. worldsteel.org/trends_indicators/

[2] J.E. ReHDER, I\&SM, August (1999) 23-27.

[3] H. Bertuing, ISIJ Int. 39 (1999) 617-624.

[4] Direct From MIDREX, First Quarter 2000; 1999 World DRI Statistics.

[5] J. Becerra, T. Guerra y F. Negrete, I\&SM, July (1999) $37-43$.

[6] G.D. Hughes, G.E. Metius y S.C. Montague, EAF Conference Proc. Vol. 58, ISS, Orlando, EE. UU., pp. 681689.

[7] A. Chatterjee, Met. Trans. 14B (1983).

[8] J. BORLEE, D. STEYLS y R. MUNNIX, 1998 ICSTI/Ironmaking Conference. Proc., Vol. 57, 1998, ISS, Toronto, Canada, pp. 869- 875 .

[9] K.L. Money, R.H. Hanewald y R.R. Bleakney, EAF Conference Proc., Vol. 58, ISS, Orlando, EE. UU. pp. 547. 560.

[10] F.G. RinkeR y V.R. DAIGA, 58 th EAF Conference Proc., Vol. 58, 1999, ISS, Chicago EE. UU., 375-380.

[11] Y. SAWA et. al, EAF Conference Proc., Vol. 58, ISS, Orlando, EE. UU., 2000, pp. 507-517.

[12] R.J. FrueHAN, Direct Reduced Iron-Technology and Economics of Production and Use, ISS, J. Feinmann-D. MacRae (Eds.), 1999, p. 165.

[13] G. LeONARD, 1999 Ironmaking Conference Proc., vol 58, Chicago, EE. UU., 1999, pp. 355-360.

[14] J. SCHENNK et. al. International conference on the Science and Technology of Ironmaking (ICSTI), Toronto, Canadá, 1998, pp. 1549-1557

[15] P. BATES, ATSE Symposium, 1998.
[16] R.J. Dry, C.P. Bates y D.P PRICE, 1999 Ironmaking Conference Proc., Chicago, Ill, EE.UU., 1999, pp. 361-366.

[17] G. Rath, H.J. LehmKuhler, G. Cavallo y O. Metelmann, 1997 Electric Furnace Conference Proc., Vol. 55, pp. 233-245.

[18] G. Hoffman, K. Shoop y K. TokUdA, 1999 Ironmaking Conference Proc., Vol. 58, 1999, pp. 367-373.

[19] G.E. Hoffman, EAF Conference Proc., ISS, Orlando, EE. UU., 2000, pp. 751-767.

[20] R.J. Fruehan, Direct Reduced Iron-Technology and Economics of Production and Use, ISS, EE. UU., 1999J. Feinmann-D. MacRae (Eds.), pp. 165.

[21] Y. Song, 1998 ICST/Ironmaking Conference Proc., vol 57, 1998, Toronto, Canada, pp. 85-92.

[22] L.K. ChIANG, Z. Ming y L. Kong, 1998 ICST/Ironmaking Conference Proc.-vol 57, 1998, Toronto, Canada, pp. 1567 1580.

[23] H.B. Lungen y R. StefFen, MPT International, 5, 1998, 58-66.

[24] J.T. KOPFLE, MPT International, 5, 1998, 67-68

[25] J.L. MAnzo, La Jornada, 25 Octubre, 2000.

[26] P.E. DUARTE, et. al., International Conference on the Science and Technology of Ironmaking (ICSTI), Vol. 57, Toronto, Canadá, 1998, p. 891

[27] A. Chatterjee, Proc. XVth Symp. SIDOR, Puerto Ordaz, Venezuela, 1988.

[28] R.H. WHIPP, Direct reduced iron-technology and economics of production and use", ISS, EE. UU. J. Feinmann-D. MacRae (Eds.), 1999, 207-223.

[29] A. ChatterjeE, Beyond the Blast Furnace, CRC press, EE. UU., 1994, p.162.

[30] B. SARMA y R.J. Fruehan, International Conference on the Science and Technology of Ironmaking (ICSTI), Vol. 57, Toronto, Canadá, 1998, pp. 1537-1548.

[31] R.J. FRUEHAN, International conference on the Science and Technology of Ironmaking (ICSTI), Vol. 57, Toronto, Canadá, 1998, pp. 59-66. 